\title{
APPLICATION OF ANTIBIOTICS AND PROBIOTICS FOR PREVENTION OF ANTIBIOTIC-ASSOCIATED DISBIOSIS IN PATIENTS WITH GENERALIZED PERITONITIS AND ENTERAL DYSFUNCTION SUPPORTS STAFF AWARENESS
}

DOI: 10.36740/WLek202103123

\author{
Viktor P. Polyovyy' ${ }^{1}$, Ruslan I. Sydorchuk', Larysa Ya. Fedonyuk², Oleksand V. Rotar', Pavlo V. Polyovyy', \\ Ilya G. Chepega' ${ }^{1}$, Alexandr A. Fomin ${ }^{3}$ \\ ${ }^{1}$ BUKOVINIAN STATE MEDICAL UNIVERSITY, CHERNIVTSI, UKRAINE \\ ${ }^{2}$ TERNOPIL NATIONAL MEDICAL UNIVERSITY NAMED AFTER I.YA. GORBACHEVSKY, TERNOPIL, UKRAINE \\ ${ }^{3}$ VINNITSYA MEMORIAL PYROGOV NATIONAL MEDICAL UNIVERSITY, VINNYTSIA, UKRAINE
}

\begin{abstract}
The aim: To clarify the efficacy of probiotics use as a preventive measure for post-antibiotic treatment in acute peritonitis and increase staff awareness related to antibiotic and probiotic use.

Materials and methods: The study design included determination of the proper antibiotic and probiotic strain combination and clinical application of probiotic strains. The control group consisted of $63(48.46 \%)$ patients who underwent traditional multimodal treatment of peritonitis and the study group of 67 (51.54\%) individuals, with inclusion of different antibiotic/probiotic combinations.

Results: Prior to antimicrobial therapy $67.7 \%$ patients of both groups'patients had severe dysbiosis, proving dysbiosis as a sign of peritonitis. S. boulardii showed widest resistance spectrum and was used for probiotic therapy in study group. Intestinal dysbiosis grades distribution in control group significantly worsened, while in study group ratio of severe dysbiosis significantly dropped from $58.2 \%$ to $38.8 \%$ with significant growth of grade Il dysbiosis to $61.2 \%$. No visible differences in disease course and clinical picture, duration or complications rate between study and control groups were observed.

Conclusions: Most of probiotic strains lack antibacterial resistance that makes meaningless their use during systemic antibiotic therapy of acute peritonitis. It is characterized by harsh changes of intestinal microbiota (severe intestinal dysbiosis). While probiotic strains showed antibiotic tolerance, their use presented no significant clinical efficacy, though high level of positive influence on intestinal dysbiosis was observed.
\end{abstract}

KEY WORDS: peritonitis, treatment, antibiotics, probiotics, physiology, microbiota

Wiad Lek. 2021;74(3 p.l):508-511

\section{INTRODUCTION}

Acute peritonitis (AP) is a comparatively common, but deadly condition, generally described as an acute inflammation of the peritoneum resulting mainly from the bacterial infection as well as other causes such as chemicals, irradiation, and foreign-body injury $[1,2]$. It is a major contributor to non-trauma deaths despite improvements in diagnosis and surgical and intensive care management. Influence on the peritoneal lining by any of these agents can lead to an inflammatory response, known as AP [3]. The structure of the etiological causes leading to AP consists of sources in the colon (32\% of patients), appendix (31\%), stomach/duodenum (18\%), small bowel (13\%), or biliary tract (6\%). About $78 \%$ patients with AP present with generalized peritonitis and $26 \%$ with severe peritonitis. The overall mortality rate significantly decreased (15-25\%) over last decades but remains high [4-7].

Multiorgan failure, or multiorgan dysfunction syndrome (MODS) is essential for peritonitis and abdominal sepsis (AS) and is observed in the vast majority of such patients [8]. The role of endotoxemia in the pathogenesis of multiorgan failure is confirmed by data on the homogeneity of systemic changes in different pathologies, which leads to AP and AS, as well as the lack of direct correlation between the type of pathogen and the nature of the disease [9-11]. Moreover, the understanding of the role of bacteremia in the pathogenesis of AS has changed radically. At the present stage, bacteremia is no longer considered as the only decisive component in the diagnosis of abdominal sepsis. It is the high concentration of endotoxins and exotoxins of bacterial cells in the blood that becomes the main factor in the activation of mediator systems, in particular cytokines $[12,13]$. Among them, TNF, interleukins, system of complement, and interferons play an important role. Damage to the epithelium in the target organs under the influence of mediators leads to significant functional disorders and forms MODS, which is similar to many inflammatory and immune dependent conditions and considered to have strong genetic background [14-16].

Development of intestinal insufficiency as a part of MODS is often underevaluated, whilst playing an extremely important 
role in the pathogenesis of peritonitis and abdominal sepsis. Under the influence of inflammatory mediators, hypercatabolism, impaired coagulation, systemic and visceral blood flow disorders, damage of enterocytes develops rapidly, almost all functions of the digestive tract are disrupted - barrier, metabolic, immunoreactive, endocrine, etc. Intestinal insufficiency is a key moment in the development of the "vicious" circle in AP and AS, because the translocation of microorganisms and their toxins supports the general inflammatory reaction, aggravating metabolic disorders [17-19].

Intestinal dysbiosis is known to be either a background or aggravating factor for multiple conditions including chronic and acute inflammatory processes, inflammatory bowel disease, non-alcoholic fatty liver disease, several types of tumors, etc. Possible mechanisms of microbiota involvement in their pathogenesis are complex and not clearly understood, though immune, metabolic and even genetic factors associated with intestinal microbiota are important in many diseases [20,21].

Relationship between virulence factors of pathogens and host resistance explains diverse clinical picture of AP and formation of AS in many patients. Furthermore, existing multimodal treatment approach including massive antimicrobial therapy seems to partially add to both developments of intestinal insufficiency and MODS. However, linkage of antibacterial therapies and intestinal insufficiency, including intestinal microbiota changes and translocation is not clearly understood. Moreover, there is related issue of insufficient staff awareness related to antimicrobial therapy application.

\section{THE AIM}

The aim of the study was to clarify the efficacy of possible use of different probiotic compositions as a preventive measure for post-antibiotic treatment in acute peritonitis and increase staff awareness related to antibiotic and probiotic use.

\section{MATERIALS AND METHODS}

Design of the study included two consecutive components, determination of the proper antibiotic and probiotic strain combination and clinical application of probiotic strains aimed on finding the probiotic activity in patients undergoing treatment. According to the aim of the study, the examination was conducted of 130 patients with acute surgical diseases of the abdominal cavity the course of which was complicated by the development of different forms of peritonitis. The study was conducted in accordance with the principles of the Council of Europe Convention on Human Rights and Biomedicine, Declaration of Helsinki on the ethical principles for medical research involving human subjects, and other valid international and national legislations in bioethics (including GCP, EU directives, etc. The study protocol was approved by the institutional ethics committee. All patients signed an informed consent prior to participating in the study.

Among study individuals, there were 73 men (56.15\%) and 57 women (43.85\%), indicating frequency of AP in both men and women. The age-related division of AP occurrence: in age categories from 40 to 60 years (41.5\%), from 61 to 80 years $(28.46 \%)$, from 20 to 40 years $(23.85 \%)$. The number of patients with concomitant somatic pathology (71 patients, $54.62 \%$ ) significantly exceeded the number of patients without comorbidities (45.38\%). Only surviving patients were included into the study.

All patients are divided into two groups. The control group consisted of $63(48.46 \%)$ patients who underwent traditional comprehensive multimodal treatment of peritonitis $[4,17,22]$ and patients of the study group consisting of $67(51.54 \%)$ individuals, who underwent multimodal treatment with inclusion of different antibiotic/probiotic combinations. Distribution of patients with peritonitis is presented in Table I.

The peculiarities of the probiotics' and antibiotics' action on the microflora were studied according to the standard microbiological methods: a culture of a probiotic strain was previously grown from a registered biological product and a suspension of $10^{9} \mathrm{CFU} / \mathrm{ml}$ was made from it using an optical standardization approach. In this study we tested following strains of probiotic microorganisms: Esherichia coli strain M-17 (Bifikol, Biofarma, Ukraine); Bacillus subtilis 3, Bacillus licheniformis 31 (Biosporin, Biofarma, Ukraine); Bacillus clausii (Enterogermina, Sanofi, France); Lactobacillus fermentum 90 TC-4 (Lactobacterin, Biofarma, Ukraine); Lactobacillus acidophilus, Lactobacillus bifidus, Lactobacillus bulgaricus, Streptococcus thermophilus (Canadian yoghurt, Astrapharm, Ukraine), Lactobacillus rhamnosus R0011, Lactobacillus rhamnosus R0049, Streptococcus thermophilus, Lactobacillus debrueckii spp. bulgaricus (Yoghurt Rosell, Astrapharm, Ukraine) Saccharomyces boulardii (Enterol 250, Biocodex, France), Enterococcus faecium (Bifiform, Ferrosan AS, Denmark), Lactobacillus GG (Bifiform Baby, Ferrosan AS, Denmark), and Aerococcus viridans (A-bacterin, Biolik, Ukraine). Sensitivity of probiotic strains to chemotherapeutic agents was studied by disc-diffusion (growth retention area size in $\mathrm{mm}$ ) method on dense medium using commercially available standard disc sets according to their manuals. Dysbiosis severity was determined in colonic content by standard microbiologic methods, and calculated as a $1 / 3$ deviation steps (grades I-III) from normal microbiota values, where 1/3 deviation was grade I, $2 / 3$ deviation was grade II and more than that was grade III. Microsoft Excel 365 and Statsoft Statistica 7.0 (USA) software packages were used for data collection and statistical analysis.

\section{RESULTS}

Probiotic strains of L. fermentum 90TS-4 and S. boulardii have been shown to be antagonistic to Enterobacteria, Pseudomonas, Staphylococci, Proteus and Bacilli, while Enterol 250 is resistant to most antibiotics. Obtained study results are presented in Tables II-IV, while summary of compatible antibiotic and probiotic combinations is shown in Table $\mathrm{V}$.

E. coli M-17, A. viridans, B. subtilis 3, B. licheniformis 31, B. Clausii, and L. fermentum 90 TC-4 showed insufficient resistance to antibiotics applying choice limits on their use and alleviating possibilities for successful systemic antimicrobial therapy of peritonitis. Probiotic strains 
included in Bifiform (E. faecium) and lactose-containing drugs (Lactobacterin, Bifiform Baby, Canadian yoghurt and Roselle yoghurt) are sufficiently resistant to cephalosporins, so they can be used in combination with these drugs. As shown at Table V, S. boulardii seems to be a better choice for use in patients undergoing systemic antibiotic treatment.

Prior to antimicrobial therapy, $75(67.7 \%)$ patients of both groups' patients had severe dysbiosis (grade III). Thirty-six (48.0\%) of them were in control group, the rest $39(52.0 \%)$ belonged to the study group. Grade II had 46 (35.4\%) and grade I - $9(6.9 \%)$ patients. Distribution of grades I-II between both groups was similar (grade II - 25 and 21 patients in study and control groups, respectively), grade I - 3 in study group and 6 in control. No significant correlation of dysbiosis and peritonitis severity before treatment was observed in both groups.

As $S$. boulardii showed widest possible resistance for most of tested antibiotics, it was used for probiotic therapy in study group patients in the form of Enterol-250, $500 \mathrm{mg}$, twice a day for seven days as a part of early enteral feeding approach.

After the treatment, intestinal dysbiosis grades distribution in control group significantly worsened. No patients had grade I dysbiosis, and $13(20.6 \%)$ had grade II, twice as low compared to the period before the antimicrobial therapy. The majority of control group patients (50 or 79.4\%) had grade III dysbiosis after antibiotics use. Study group patients after combined use of antibiotics and selected probiotic demonstrated absence of grade I dysbiosis, too. However, ratio of severe dysbiosis (grade III) significantly dropped from $58.2 \%$ to $38.8 \%$ (26 patients) with significant growth of grade II dysbiosis to $61.2 \%$ (41 patients). No visible differences in disease course and clinical picture, as well as duration of treatment or complications rate between study and control groups were observed in this study.

\section{DISCUSSION}

Acute peritonitis and its generalized forms often lead to systemic changes defined as abdominal sepsis (AS) and multiple organ dysfunction syndrome (MODS). While antibiotic use is an essential part of peritonitis managements it may under these circumstances have negative influence, too. This is exactly one of the reasons why the vast majority of hospitals employ different programs for increasing staff awareness towards antibiotic use as an addition to more common antibiotic resistance awareness. However, very few of these programs including globally spread initiatives or organizations like APUA (Alliance for the Prudent Use of Antibiotics) pay attention for the alleviation of the antibiotic use side effects, focusing mainly on preventing unnecessary use only [23]. Comparatively few papers published under the auspices of APUA deal with inter-bacterial relationships as a part of understanding antibiotic resistance [24].

Multiple genetic studies of both intestinal microbiome and proteome, show existence of complex cross-related links between human genomic single nucleotide polymorphisms (SNP) and occurrence of different conditions serving as a background for AS and MODS. Moreover, there are ties connecting intestinal microbiome and human genome. Interestingly, connections of several SNPs, metabolic and endothelial changes are well associated with changes in gut microbiota and vice versa [25]. Such complex interrelations make situation even more complex. While influence of antibiotics on intestinal microflora seems to be well established, the use of probiotics to alleviate negative influence of antibacterial therapy remains generally confusing. There are both supporting and challenging reports on this issue, especially concerning acute conditions like AP and AS [26].

In this study we attempted to add clarification on the problem while finding out the appropriate combination of antibiotic and probiotic to avoid preliminary elimination of probiotic strain by the drug used. In addition, both clinical and microbiologic efficacy of such approach were evaluated. Our findings support the idea, that insufficient efficacy of the probiotic use in AP may be associated with inappropriate selection of probiotic strain and its elimination or neutralization by the applied antibiotic itself. But even when appropriate antibiotic and probiotic composition is used, whilst there is a good impact on microbiota's balance as shown by better dysbiosis grades proportions compared to control, no reliable support in terms of clinical picture or complication rates was observed. It rises multiple questions concerning the possible mechanisms of microflora involvements into the acute inflammatory process, metabolic and immune changes and AS/MODS formation during acute peritonitis. Further study of these mechanisms may produce sufficient data to understand why clinical efficacy of probiotic and antibiotic use is confusing, while being effective in correction of dysbiosis itself. Furthermore, this study supports awareness of medical personnel towards the proper use of antibiotics.

\section{CONCLUSIONS}

Most of probiotic strains lack antibacterial resistance that makes meaningless their use during systemic antibiotic therapy of acute peritonitis. Acute peritonitis is characterized by harsh changes of intestinal microbiota as proved by severe intestinal dysbiosis registered in this study. While selected probiotic strains of $S$. boulardii showed good antibiotic tolerance when combined with common antimicrobial drugs, their use presented no significant clinical efficacy, though high level of positive influence on intestinal dysbiosis was observed.

\section{REFERENCES}

1. Tochie J.N., Agbor N.V., Frank Leonel T.T. et al. Global epidemiology of acute generalised peritonitis: a protocol for a systematic review and meta-analysis. BMJ Open. 2020;10(1):e034326.

2. Riché F.C., Dray X., Laisné M.J. et al. Factors associated with septic shock and mortality in generalized peritonitis: comparison between communityacquired and postoperative peritonitis. Crit Care. 2009;13(3):R99.

3. Sartelli M., Abu-Zidan F.M., Labricciosa F.M. et al. Physiological parameters for Prognosis in Abdominal Sepsis (PIPAS) Study: a WSES observational study. World J Emerg Surg. 2019;14(34)..

4. Gauzit R., Péan Y., Barth X. et al. Mistretta F, Lalaude 0; Top Study Team. Epidemiology, management, and prognosis of secondary non-postoperative peritonitis: a French prospective observational multicenter study. Surg Infect (Larchmt). 2009;10(2):119-27. 
5. Ivashchuk S.I., Sydorchuk L.P. The parameters of liver functional state as a risk factor of edematous pancreatitis development providing of genetic determination of IL-4 production. Wiadomosci lekarskie (Warsaw, Poland: 1960). 2019;72(4):639-644.

6. De Simone B., Ansaloni L., Sartelli M. et al. The Operative management in Bariatric Acute abdomen (OBA) Survey: long-term complications of bariatric surgery and the emergency surgeon's point of view. World J Emerg Surg. 2020;15(2).

7. Chromik A.M., Meiser A., Hölling J. et al. Identification of patients at risk for development of tertiary peritonitis on a surgical intensive care unit. J Gastrointest Surg. 2009;13(7):1358-67.

8. Abaziou T., Vardon-Bounes F., Conil J.M. et al. Outcome of communityversus hospital-acquired intra-abdominal infections in intensive care unit: a retrospective study. BMC Anesthesiol. 2020;20(1):295.

9. SydorchukL., DzhuryakV., SydorchukA. et al.The cytochrome 11B2 aldosterone synthasegeners 1799998 singlenucleotide polymorphism determines elevated aldosterone, higher blood pressure, and reduced glomerular filtration, especially in diabetic female patients. Endocrine Regulations. 2020;54(3):217-226.

10. Sydorchuk L.P., Dzhuryak V.S., Sydorchuk A.R. et al. Association of lipids' metabolism disorders with aldosterone synthase CYP11B2 (-344C/T) gene polymorphism in hypertensive patients depending on glomerular filtration rate. Pharmacology0nLine. 2020;2:230-242.

11. Hameed T., Kumar A., Sahni S. Et al. Emerging Spectrum of Perforation Peritonitis in Developing World. Front Surg. 2020;7:50.

12. Sydorchuk L.P., Syrota B.S., Sydorchuk A.R. et al. Clinical markers of immune disorders in the pathogenesis of Escherichia coli enteritis. Arch Balk Med Union. 2019;54(1):89-96.

13. Yoon Y.K., Kim J., Moon C. Et al. Antimicrobial Susceptibility of Microorganisms Isolated from Patients with Intraabdominal Infection in Korea: a Multicenter Study. J Korean Med Sci. 2019;34(47):e309.

14. Tochie J.N., Agbor N.V., Frank Leonel T.T. et al. Global epidemiology of acute generalised peritonitis: a protocol for a systematic review and meta-analysis. BMJ Open. 2020;10:e034326.

15. Sheremet M.I., Sydorchuk L.P., Shidlovskyi V.O. et al. Association of the blood serum cytokines' rate and lymphocytes' apoptosis with polymorphic variants of the BCL-2 (Rs17759659), CTLA-4 (rs231775) and APO-1/FAS (rs2234767) genes in patients with nodular goiters in autoimmune thyroiditis and thyroid adenoma. Romanian Journal of Morphology and Embryology. 2017;58(3):931-939.

16. Sydorchuk L.P., Serdulets Y.I., Sydorchuk A.R. et al. The polymorphism of matrilin-3 (rs77245812) and interleukin-10 (rs1800872) genes in osteoarthritis patients with arterial hypertension, obesity and type 2 diabetes mellitus. Arch Balk Med Union. 2017;52:422-429.

17. Membrilla-Fernández E., Sancho-Insenser J.J., Girvent-Montllor M. et al. Secondary Peritonitis Spanish Study Group. Effect of initial empiric antibiotic therapy combined with control of the infection focus on the prognosis of patients with secondary peritonitis. Surg Infect(Larchmt). 2014;15(6):806-14.

18. Sydorchuk L.I., Hrushko 0.I., Sydorchuk I.Y. et al. Early changes of luminal colonic microbiome in experimental abdominal sepsis. Infection. 2019;47(Suppl. 1):5.

19. Maseda E., Ramírez S., Picatto P. et al. HELP Investigators and the Perioperative Infection Research Group. Critically ill patients with community-onset intraabdominal infections: Influence of healthcare exposure on resistance rates and mortality. PLoS One. 2019;14(9):e0223092.

20. Lobo L.A., Benjamim C.F., Oliveira A.C. The interplay between microbiota and inflammation: lessons from peritonitis and sepsis. Clin Transl Immunology. 2016;5(7):e90.
21. Fay K.T., Klingensmith N.J., Chen C.W., et al. The gut microbiome alters immunophenotype and survival from sepsis. FASEB J. 2019;33(10):11258-11269.

22. Doklestić S.K., Bajec D.D., Djukić R.V. et al. Secondary peritonitis evaluation of 204 cases and literature review. J Med Life. 2014 Jun 15;7(2):132-8.

23. O'Brien T., Stelling J. Integrated multilevel surveillance of the world's infecting microbes and their resistance to antimicrobial agents. Clin. Microbiol. Rev. 2011;24(2):281-295.

24. Garbeva P., Silby M.W., Raaijmakers J.M. et al. Transcriptional and antagonistic responses of Pseudomonas fluorescens Pf0-1 to phylogenetically different bacterial competitors. ISME J. 2011;5:973985.

25. Sydorchuk L.P., Sokolenko A.A., Sydorchuk A.R. et al. Insulin resistance in patients with arterial hypertension and abdominal obesity depending on ACE and PPAR- $\gamma 2$ genes polymorphism: A new opinion concerning an old problem. New Armenian Medical Journal 2015; 9:43-51.

26. Barnett R.E., Conklin D.J., Ryan L. et al. Anti-inflammatory effects of miR-21 in the macrophage response to peritonitis. J Leukoc Biol. 2016;99(2):361-371.

The research was performed as part of the initiative research work "Clinical and experimental substantiation of prevention and individualized staged treatment of purulent-septic complications of acute abdominal surgical pathology", the state registration number: 117 U002356.

\section{ORCID and contributionship:}

Victor P. Polyovyy: 0000-0002-4345-9802 A, E, F

Ruslan I. Sydorchuk: 0000-0002-3603-3432 ${ }^{A, D, F}$

Larysa Ya. Fedoniuk: 0000-0003-4910-6888 C, E, F

Oleksandr V. Rotar: 0000-0002-9434-0377 B, E

Pavlo V. Polyovyy: 0000-0002-1250-0366 E,F

Ilya G. Chepega: 0000-0002-3861-7538 B,C

Alexandr A. Fomin: 0000-0002-0420-4655 B,D

\section{Conflict of interest:}

The Authors declare no conflict of interest

\section{CORRESPONDING AUTHOR Victor P. Polyovyy \\ Chief of General Surgery Department \\ Bukovinian State Medical University \\ Teatralna square, 2, 58002 Chernivtsi, Ukraine \\ tel.: +380954132678 \\ e-mail: rsydorchuk@bsmu.edu.ua}

Received: 15.10 .2020

Accepted: 04.02 .2021

A - Work concept and design, B - Data collection and analysis, C - Responsibility for statistical analysis, D-Writing the article, $\mathbf{E}-$ Critical review, $\mathbf{F}-$ Final approval of the article 